\title{
In silico evaluation of isatin-based derivatives with RNA- dependent RNA polymerase of the novel coronavirus SARS-CoV-2
}

Rajesh Kumar $\mathbf{M}^{\dagger 1}$, Daniel A. Gideon ${ }^{\dagger 2}$, Richard Mariadasse ${ }^{\dagger 3}$, Vijay Nirusimhan ${ }^{2}$, Sherlin Rosita $\mathrm{A}^{2}$, Jesu Castin $\mathbf{E}^{2}$, Jeyakanthan $\mathbf{J}^{3 *}$ and Violet Dhayabaran $\mathbf{V}^{1 *}$

Author contributions:

Note: - these authors contributed equally

RKM synthesized the compounds (manuscripts published earlier) and discovered that these compounds possess antiviral activity

DAG planned the work, interpreted the data, prepared images and wrote the paper

RM Performed the dynamics work and wrote the results for MD simulations and DFT calculations

VN performed the docking work

SR and JC analyzed results and made some of the images \& tables and edited the paper draft

VVD and JJ conceived the concept, provided infrastructure, analyzed the results and gave critical inputs to bring the manuscript to its final shape.

* denotes the corresponding authors

Dr. V. Violet Dhayabaran

Associate Professor of Chemistry and Dean of Sciences,

Bishop Heber College (Autonomous),

Vayalur Road, Tennur, Tiruchirappalli-620017.

(Affiliated to Bharathidasan University),

Tiruchirappalli, Tamil Nadu, India.

Email: violet.bhc@gmail.com; violetdhaya@gmail.com

Dr. J. Jeyakanthan

Professor and Head,

Department of Bioinformatics,

Alagappa University,

Karaikudi, Tamil Nadu, India.

Email:jjkanthan@gmail.com

Authors' affiliation addresses

1 Department of Chemistry, Bishop Heber College (Autonomous), Vayalur Road, Tiruchirappalli-17.

${ }^{2}$ Department of Biotechnology \& Bioinformatics, Bishop Heber College (Autonomous), Vayalur Road, Tiruchirappalli-17.

${ }^{3}$ Department of Bioinformatics, Alagappa University, Karaikudi, Tamil Nadu, India. 


\section{Abstract}

Isatin (1H-indole-2,3-dione)-containing compounds have been shown to possess several remarkable biological activities. We had previously explored a few isatin-based imidazole derivatives for their predicted dual activity against both inflammation and cancer. We explored 47 different isatin-based derivatives (IBDs) for other potential biological activities using in silico tools and found them to possess anti-viral activity. Using AUTODOCK tools, the binding site, binding energy, inhibitory constant $/ K_{\mathrm{i}}$ and receptorligand interactions for each of the compounds was analyzed against SARS-CoV-2 RNAdependent RNA polymerase $(\mathrm{RdRp})$. The partition coefficient $(\log \mathrm{P})$ values were predicted using MedChem Designer tool. Based on the best $K_{\mathrm{i}}$, binding energy and the ideal range of $\log \mathrm{P}$ (between 1.0-3.0), 10 out of total 47 compounds were deemed to be prospective RdRp inhibitors. Some of these compounds gave better $K_{\mathrm{i}}$, binding energy and $\log \mathrm{P}$ values when compared to standard $\mathrm{RdRp}$ inhibitors such as remdesivir $\left(K_{\mathrm{i}}=15.61 \mu \mathrm{M}, \log \mathrm{P}=2.2\right.$; binding energy $=-6.95$ ), a clinically approved RdRp inhibitor which is widely used for critical care of COVID-19 patients. The same in silico parameters were assessed for 9 other popular RdRp inhibitors (other than remdesivir), which were earlier used to target RdRp of other viruses, and are now repurposed to target SARS-CoV-2 RdRp. The results showed that the 10 selected isatin-based derivatives (IBDs) could be further explored for activity against SARS-Cov-2. In the present study we evaluated the efficacy of these compounds in silico.

\section{Keywords}

SARS-CoV-2, RNA dependent RNA polymerase (RdRp), isatin-based derivatives (IBDs), remdesivir, docking 


\section{Abbreviations}

BAL - Balapiravir; FAV - Favipiravir; GAL - Galidesivir; IBDs - Isatin-based derivatives; MERS - Middle East Respiratory Syndrome; nsp - non-structural proteins; RdRp - RNAdependent RNA polymerase; REM - remdesivir; RIB - Ribavirin; SARS- Severe Acute Respiratory Syndrome; SET- Setrobuvir; SOF - Sofosbuvir; TEN - Tenofovir

\section{Introduction}

SARS-CoV-2 mediated COVID-19 is a currently active global pandemic with 79.4 million registered cases of infection worldwide and mortality of 17,43,760 seriously affected individuals globally, as per the latest data from the World Health Organization on December 25th, 2020. In close to a year from the time of outbreak in December 2019 in Wuhan city, Hubei province of China, SARS-CoV-2-mediated COVID-19 disease has progressed to become one of the most perplexing health conditions in the history of mankind. SARS-CoV-2 is a positive-sense, enveloped virus of the coronaviridae family which causes coagulopathy, elevated D-dimer levels and severe respiratory distress/pulmonary failure leading to pneumonia and eventually, death (Li et al., 2020). While countries such as United States, Brazil, Russia, India and the United Kingdom have the highest recorded infection rates globally, the mortality numbers are the highest in the United States, Brazil and Italy (Yang et al., 2020). Several countries and pharmaceutical companies are actively engaged in discovering vaccines and therapeutic drugs which can effectively combat COVID-19 (Liu et al., 2020).

SARS-CoV-2 possesses a linear Single Stranded (Chen et al.) RNA genome ( $30 \mathrm{~kb}$ or higher), comprising of 14 open reading frames (ORFs) which encode for 27 proteins; there are 4 key structural proteins such as S (spike), M (matrix), E (envelope) and N (nucleocapsid) as well as 15 non-structural proteins (nsps) (Wu et al., 2020). Using its spike protein, the 
virus binds to human angiotensin-converting enzyme (ACE2) receptors and gains entry into cells. The gene $n s p 12$ encodes for RNA-dependent RNA polymerase (RdRp), a key enzyme responsible for replication of RNA from an RNA template; also, protein synthesis occurs from the RNA genome to form structural as well as non-structural proteins. Blocking the RdRp protein can inhibit the transcription of both structural and non-structural viral proteins. Thus, $\mathrm{RdRp}$ represents a viable and attractive target for inhibiting viral proliferation and transcription of genes encoded by the viral genome. Gao et al. had reported the structure of RdRp (nsp12) of the novel beta-coronavirus SARS-CoV-2, which can bind with two of its protein cofactors, nsp7 and nsp8 at $2.9 \AA$ resolution (Gao et al., 2020). Standard RdRp inhibitors such as remdesivir (REM), sofosbuvir (SOF), galidesivir (GAL), balapiravir (BAL), tenofovir (TEN), IDX-184 (IDX), YAK (Ahmad et al.), setrobuvir (SET), favipiravir (FAV) and ribavirin (RIB) are known to be potent coronavirus RdRp inhibitors (Elfiky, 2020). Apart from these standard and well-researched drugs, newer classes of RdRp inhibitors with much greater inhibitory potential are being researched. Since RdRps of several viruses such as coronaviruses (SARS, MERS etc.), Hepatitis C virus (HCV), Dengue virus, Ebola virus and Zika virus are known to share structural similarities, inhibitors which were discovered against Ebola RdRP can be used against SARS-CoV-2 RdRp. Therefore, through drug repurposing, several drug molecules which were earlier used for the treatment of other viral diseases are now being tested against various proteins targets of SARS-CoV-2especially, RdRp (Wu et al., 2020). In this context, it is important for the scientific community to explore new drug candidates to act as binding-based inhibitors of SARS-CoV2 proteins and increase our repertoire of drugs which could effectively combat COVID-19 disease. Since there are speculations about long-term immunity from vaccine candidates which have been successfully cleared for human use, there is a need for new classes of 
therapeutic small molecules which could alleviate the symptoms of COVID-19 and also cure the disease.

Herein, we report the in silico efficacy of 47 synthetic IBDs against RdRp of SARSCoV-2 and discuss the possibility of exploring the best drug candidates in the near future for their potential use against COVID-19 disease.

\section{Materials and Methods}

\section{Ligand and Protein preparation}

Based on the literature survey, some of RdRp inhibitors (control drugs) were identified and their structures were derived from PubChem database by retrieving the canonical smiles. The chemical properties of these compounds (control drugs \& IBDs) were identified using MedChem Designer which aids in the prediction of S+logP, S+logD, TPSA, Lipinski's Rule of Five, MlogP and Differentiate coefficient. The target macromolecule (RdRp) was downloaded from Protein Data Bank (PDB ID: 7BTF), whose crystal structure (with the resolution of $2.95 \AA$ ) was published by Gao et al. (Gao et al., 2020). After removal of water molecules, the existing ligands (HETATM), cofactors and chelators were removed to prepare the target receptor. The SMILES of the 10 control and 47 IBDs were converted using the online SMILES translator tool into .pdb format and the structures of these compounds were drawn using CHEMDRAW.

\section{Molecular Docking}

The docking studies were performed to identify the molecular interactions between the 3D model of RdRp (PDB ID: 7BTF) and chemical compounds (both control drugs as well as isatin based imidazole derivatives) using MGL tools (AutoDock 4.2) (Goodsell et al., 1996). Both the RdRP receptor and small molecule ligands were converted into PDBQT 
format from PDB file format by following the standard AutoDock protocol. AutoGrid was adjusted and the dimensions of XYZ were set at 60 × 60 × $60 \AA^{3}$ respectively. The spacing angstroms were set at $0.375 \AA$ and Gasteiger charges and polar hydrogen atoms were added to the target (RdRP macromolecule). Prior to the commencement of docking, the active torsions and torsional degree of freedom for small molecules were optimized. The Genetic Algorithm (GA)-Lamarckian principle was used and the program was set to complete 10 docking runs. The potency of the inhibitors was gauged based on binding energy (Gibbs free energy, $\Delta \mathrm{G}$ ) values ( $\mathrm{kcal} / \mathrm{mol})$. PyMoL, a molecular visualization tool, was used to view the interactions between the target $\&$ small molecules and to measure the distance (bond length) for the docked complex. The best output poses were analysed for interactions between the receptor and ligand. The $2 \mathrm{D}$ poses of the best hits for each compound was generated using Accelrys Discovery Studio Visualizer 2.5 (Visualiser).

\section{Molecular Dynamics Simulation (MDS) studies}

The native structure and ligand-bound complexes (top five docking score compounds and Remdesvir molecule) of RdRp were subjected to molecular dynamics simulation studies (MDs) using GROMACS (GROningen MAchine for Chemical Simulations) v5.1 software (Ahmad et al., 2020). The GROMOS53a5 force field was employed to simulate both the native structure and bound complexes of RdRp enzyme. The ligands topologies were generated using PRODRUG server (http://davapc1.bioch.dundee.ac.uk/programs/prodrg) and it was incorporated into the RdRp structure to perform MDS studies. The cubic box was set with $1 \mathrm{~nm}$ distance from the molecule and the systems (native structure and ligand-bound complexes) were solvated with SPC216 water model. Periodic Boundary Condition (PBC) was applied in all direction to minimize the edge effects. The net charge of systems was neutralized by adding $\mathrm{Na}+$ counter ions to evaluate the finite electrostatic values. Energy 
minimization was performed using Steepest Descent Algorithm (50,000 steps) with a tolerance of $1000 \mathrm{~kJ} \mathrm{~mol}^{-1} \mathrm{~nm}^{-1}$ followed by two equilibration phases; NVT (constant number of particles, volume, and temperature) and NPT (constant number of particles, pressure, and temperature) was maintained (Mariadasse et al., 2019). The Position Restraint (PR) method was employed in both NVT and NPT for 100 ps followed by conjugate gradient algorithm with a tolerance of $1000 \mathrm{~kJ} \mathrm{~mol}^{-1} \mathrm{~nm}^{-1}$. The linear constraint solver (LINCS) algorithm was customised to constrain all the bonds in the systems. A $1.2 \mathrm{~nm}$ cut-off of shortrange non-bonded interaction was calculated followed by; the long-range electrostatic interactions that were calculated with a cutoff of $0.16 \mathrm{~nm}$ using the particle mesh Eshwald (PME) method. The temperature $(310 \mathrm{~K})$ was regulated using V-rescale weak coupling method. The pressure (1 atm), density, and total energy of the system was equilibrated by Parrinello-Rahman (PR) method (Choubey et al., 2020; Mariadasse et al., 2019). All the preequilibrated systems were performed for 100,000 ps (100 ns) MDS studies.

\section{MMPBSA analysis of RdRp complexes}

The Molecular Mechanics Poisson-Boltzmann Surface Area (MMPBSA) is an effective method to understand the molecular recognition of protein-ligand, protein-protein and protein-DNA complexes. MMPBSA combined with molecular dynamics is most commonly used to calculate the binding free energies ( $\Delta$ Gbind) of protein-ligand complexes. Here, molecular dynamics simulation trajectories (different protein-ligand complexes) of last 15 ns were analysed using g_mmpbsa tool in the Gromacs software and their binding free energies were calculated by the following equation (Mariadasse et al., 2019; Murugan et al., 2020),

$$
\Delta \mathbf{G}_{\text {bind }}=\mathbf{G}_{\text {complex }}-\left(\mathbf{G}_{\text {protein }}+\mathbf{G}_{\text {ligand }}\right)
$$


Where $\Delta \mathrm{G}_{\text {complex, }} \Delta \mathrm{G}_{\text {protein }}$ and $\Delta \mathrm{G}_{\text {ligand }}$ defines the total energy, total binding free energy of protein and ligand respectively.

\section{DFT studies}

Density Functional Theory (DFT) calculation was performed for the top five compounds (Compounds-16, 24, 28, 38 and 40, which were chosen based on their $K_{i}$ and $\log$ P values) using Jaguar 9.1 version implemented in the Schrodinger software suite 2020-2. Molecular electronic properties such as HOMO (Highest Occupied Molecular Orbital) and LUMO (Lowest Unoccupied Molecular Orbital) of the compounds were accurately determined, and thereby the chemical reactivity for the inhibitory reaction was predicted (Ahmad et al., 2020; Mariadasse et al., 2019). A hybrid DFT approach (B3LYP-Becke's three-parameter exchange potential and the Lee-Yang-Parr correlation functional) was performed for geometry optimization with the basis set of $6-31 \mathrm{G}^{* *}$. The energy of the compounds at the aqueous condition was calculated by the Poisson-Boltzmann solver which yielded additional information of about the local and global indices for its reactivity (Langeswaran et al., 2018). Overall, the charge transfer mechanism for the inhibitory reaction of the compounds can be understood by analyzing the HOMO and LUMO molecular orbitals of the receptor-drug binding combinations.

\section{Results and Discussion}

\section{Chemistry of the IBDs and active groups involved in interaction with RdRp}

Our group has actively researched the medicinal chemistry of IBDs and tested their efficacy in silico and in vitro (Dileepan et al., 2018; Kumar M and Alagumuthu, 2018; Kumar et al., 2018; Rajesh Kumar et al., 2018). Isatin derivatives were shown to possess anxiogenic, anticonvulsant, sedative, antiviral, antituberculosis, anticancer and anti-allergic activities (Pandeya et al., 2005). Originally, these IBDs and spiro-oxindoles were prepared using a 
novel, one-pot synthesis approach in treatment of breast and prostate cancer. However, several methods that have been used for the synthesis and molecular characterization of the IBDs were reported elsewhere (Kumar et al., 2020; Kumar et al., 2018; Rajesh Kumar et al., 2018). Some of the compounds we had synthesized and reported earlier showed greater in vitro potency against MCF-7 breast cancer cell lines, when compared to the standard anticancer drug, doxorubicin (Kumar et al., 2020). Recently, we synthesized these compounds and showed through in vitro and in silico investigations that these IDBs possessed dual activity against inflammation and pain by blocking the action of phosphoinositide- 3 kinase (PI3K) and cyclooxygenase-2 (COX-2), respectively (Kumar et al., 2020).

Among the IBDs, compounds 1a-13a are isatin-based derivatives with thiourea acting as a bridge between the oxoindole (which is similar to isatin) and imidazolyl methyl structures and hence, have been referred to as isatin-based imidazole compounds. The synthesis as well as NMR and HR-MS characterization of isatin-based imidazole derivatives was reported in our group's recently published paper (Kumar et al., 2020). Compounds 21a29a, 21a-24a contain thiourea and the rest of the IBDs until 29a possess urea; since these drugs possess a quinoline ring, they are chemically referred to as indoline-based dihydroxy thiocarbamides (Kumar M and Alagumuthu, 2018). Molecules 30a-47a are spiro-chromeno indoline-triones with various substituents such as halides and alkyl groups (Kumar et al., 2018).

Using in silico tools such as molinspiration and PASSonline, we assumed that these 47 IBDs may also possess antiviral activity. Isatin compounds have several biological activities and are either agonists or antagonists of key cellular receptors. Isatin is present naturally in humans and is sometimes found at very high (millimolar) concentrations in some biological tissues and has been known to act as a ligand of several proteins (Medvedev et al., 2007) and hence, drugs with this group may have lower toxicity in vivo (Eggadi et al., 2013). 


\section{Binding energy and $K_{\mathrm{i}}$ values}

Using molecular docking, the binding of the IBDs was assessed against SARS-CoV-2 RdRp (PDB ID: 7BTF). In Figure 1, the structures of ten IBDs- 13a, 14a, 16a, 18a, 19a, 23a, 24a, 28a, 38a and 40a) are shown. Out of 47 molecules, these are the compounds with the best Gibbs free energy values $(\Delta \mathrm{G})$ and therefore, would bind better to RdRp. The structures of the other molecules and their properties are given in Table S1 (supporting material). These molecules were chosen based on the best binding energy, $K_{\mathrm{i}}$ and also $\log \mathrm{P}$ values after comparing them against the control RdRp inhibitors such as REM, SET, SOF and YAK. When comparing the binding energies of the six other compounds (BAL, FAV, GAL, TEN, RIB and IDX-184), REM, SET, SOF and YAK were deemed to have greater affinity to RdRp. YAK (-8.85) and SET (-8.78) had better $\Delta \mathrm{G}$ values than the other control drugs such as REM (-6.55), SOF (-6.78), GAL (-5.84), IDX-184 (-5.67), FAV (-5.25), RIB (-4.76), TEN (-4.48) and BAL (-4.13); see Table 1 and Table S1 (supporting information) for more details. When comparing the $K_{\mathrm{i}}$ values of these compounds, compounds $28 \mathrm{a}, 40 \mathrm{a}$ and $16 \mathrm{a}$ had much lower $K_{\mathrm{i}}$ values (within $\left.0.2 \mu \mathrm{M}\right)$ when compared to REM $(15.91 \mu \mathrm{M})$ and SOF $(10.75 \mu \mathrm{M})$; also, these ten isatin-based derivatives possessed better $K_{\mathrm{i}}$ values when compared to the other 37 derivatives (See Table 1 and Table S1). The difference in $K_{\mathrm{i}}$ values is remarkable because IBDs 28a, 40a and 16a have been predicted to possess almost 80 and 54 times more favorable binding energy than REM and SOF, respectively.

REM and SOF are nucleotide analogs which are known to be added to the growing RNA chain, effectively terminating RNA synthesis (Gao et al., 2020; Gordon et al., 2020b). The RNA genome of SARS-CoV-2 codes for nsps from two open reading frames (ORFs), namely ORF1a and ORF1b. ORF1a codes for a smaller polypeptide (pp1a), which has a size of $\sim 440-500 \mathrm{KDa}$ and $11 \mathrm{nsps}$ and ORF1b produces a larger polypeptide (pp1ab) which is $\sim 740-800 \mathrm{KDa}$ in size, coding for 15 nsps. Currently, remdesivir (patented by Gilead) is used 
as the drug of choice for the management of COVID-19 disease in many countries. RdRp (nsp12) contains several domains, which were illustrated by Gao et al (Gao et al., 2020). The same representation of $\operatorname{RdRp}$ (including colour) is followed in this paper, in Figure 5. REM was originally used against Ebola virus RdRp; but, through drug repurposing, it has been found to inhibit the RdRp of SARS-CoV-2. The structures of the other IBDs are shown in Table S2.

Coming from the N-terminal end, the protein contains NiRAN (nidovirus RdRpassociated nucleotidyltransferase) motif-1 (residues 4-28), $\beta$-clamp (residues 29-50), NiRAN motif-2 (amino acids 51-249), interface region (residues 250-365), fingers 1 region (366581), palm 1 region (581-620), fingers-2 domain (621-679), palm-2 region (680-815) and the thumb region (residues 816-932) (Gao et al., 2020). The RdRp active site is formed by a few conserved motifs, A-G within the palm domain, in which, motif A contains the classic divalent ion-binding conserved glutamate (D) residue at position 618. Another D residue (623) is also found in this 611-TPHLMGWDYPKCDRAM-626 motif. The catalytic residues of SDD (amino acids 759, 760 and 761) are located in motif C (753FSMMILSDDAVVCFN-767), in between two $\beta$-strands. The other key catalytic residues such as 317-GDD-319 and 327-GDD-329 are also conserved in many other viruses. REM is a prodrug in its monophosphate form (RMP), which is converted to an active triphosphate form known as remdesivir triphosphate (RTP). Upon binding to RdRp, REM exerts its RdRpinhibitory activity in a manner which is similar to nucleotide analog prodrugs and triggers non-obligate RNA chain termination (Yin et al., 2020).

Interestingly, REM binds to this critical region and is found to interact with key aspartate residues which are critical for the phosphoryl group transfer reaction and also for binding to divalent metal ions (Gao et al., 2020). The two aspartate residues of the motif $\mathrm{C}$ in the DDxVV pattern are usually conserved in coronaviruses such as MERS-CoV (Gordon et 
al., 2020a; Gordon et al., 2020b), SARS-CoV and SARS-CoV-2 and these three coronaviruses alone (amongst the coronavirus family) possess a serine residue instead of glycine, which precedes the two aspartate residues in RdRps of other viruses such as HCV (Buonaguro et al., 2020). The $E C_{50}$ of REM in Vero-E6 cells was found to be $23.15 \mu \mathrm{M}$, while homoharringtonine and emetine were much more effective, with in vitro $E C_{50}$ values of 2.55 and $0.46 \mu \mathrm{M}$, respectively, for the two compounds (Choy et al., 2020). A combination of REM and emetine proffered in vitro synergistic effect in Vero-E6 cells in the same study. While it is important to pursue active research to identify molecules with much more promising in vitro and in vivo antiviral activity, it is also vital to ensure that these compounds do not cause significant toxicity in humans. The binding energies of all the 47 IBDs and ten well-known RdRp inhibitors are presented in Figure 2. Interestingly, many of the IBDs were found to interact with $\mathrm{RdRp}$ with $\Delta \mathrm{G}$ values close to or below $-8.0 \mathrm{kcal} / \mathrm{mol}$. Most of these compounds, had stable binding interactions with $\mathrm{RdRp}$ when compared to most of the control/known RdRp inhibitors, including REM (Figure 2). Earlier, Elfiky had reported the binding energies $(\Delta G)$ of the five approved RdRp inhibitors - GAL, REM, TEN, SOF \& RIB to be $-7.0,-7.6,-6.9,-7.5$ and $-7.8 \mathrm{kcal} / \mathrm{mol}$, respectively (Elfiky, 2020). Interestingly, in that work, the physiological substrates of RdRp - riboGTP, UTP, CTP and ATP were found to possess binding energies in the range of -7.0 to $-8.7 \mathrm{kcal} / \mathrm{mol}$. This data supports our work because some of the IBDs possess comparable binding energies to the ribonucleotide substrates and therefore, can be presumed to be better ligands of RdRp than some of the commercially available drugs.

In Table S3, the properties of the 47 isatin-derived molecules such as $\log \mathrm{P}, \mathrm{M} \log \mathrm{P}$, $\mathrm{S}+\log \mathrm{P}$ and $\mathrm{S}+\log \mathrm{D}$ are shown. In medicinal chemistry and pharmacology, $\log \mathrm{P}$ is the particular ratio of distribution of the concentrations of a given solute between two solvents such as octanol and water and thus, it is a measure of hydrophobicity of the compound. A 
compound with positive values $(>0)$ is hydrophobic, while a negative score signifies hydrophilicity. MlogP, the Moriguchi octanol-water partition coefficient, is used interchangeably with $\log \mathrm{P}$ to denote the partitioning of a solute between octanol and water and is useful in quantitative structure-activity relationship (QSAR) studies (Eros et al., 2002). $\log S$, the hydrophilicity/aqueous solubility of a compound is usually in the range of -4 for $\sim 80 \%$ of commercially available drugs (which are usually hydrophobic). Although $\log \mathrm{D}$ is another descriptor for lipophilicity, it applies to ionizable compounds and better explains partitioning or distribution of a molecule/drug in body fluids and organs. $\mathrm{S}+\log \mathrm{P}$ and $\mathrm{S}+\log \mathrm{D}$ values are simulated values of $\log \mathrm{P}$ and $\log \mathrm{D}(\mathrm{MedChem}$ designer software) which are used in QSAR studies to predict the ADME properties of molecules; while $\log \mathrm{P}$ is a simpler measure of hydrophobicity, $S+\log P$ and $S+\log D$ deal with the physiological partitioning/distribution of a molecule in the human body, particularly with reference to their pKa values and ionization state.

\section{Plot of binding energy vs. $\log P$}

We prepared a $\log \mathrm{P}$ vs. binding energy plot to identify molecules with the best combined coordinates of $\log \mathrm{P}$ and binding energy. The data points were divided into four sections on a quadrant - a) low binding and higher solubility, b) high binding and higher solubility, c) high binding and lower solubility and finally, d) low binding and lower solubility. The molecules with ideal $\log \mathrm{P}$ (as well as high binding energy zone inside the green box) were reckoned to be candidates for further studies. In the blue and red zones, the drugs had much higher $\log \mathrm{P}$ values of $>3$ and $>4$, respectively. While this division is not based on any known principles of medicinal chemistry, the division of the data points into four sections, i.e., a quadrant based on solubility as well as binding energy was done to identify the molecules which can be considered for further in vitro experimentation in the near future. While REM had a much lower binding energy, it had a $\log \mathrm{P}$ value of close to 2.2, 
which is comparable to the $\log \mathrm{P}$ of some IBDs. Other drugs such as BAL, TEN, FAV and IDX had much lower binding energies (and therefore, poorer binding to RdRp) than the ten selected test IBDs presented in Figure 2 and Table S1. SOF had a binding energy comparable to that of REM, but showed a slightly lower $\log P$.

While GAL and RIB were hydrophilic, their $\Delta \mathrm{G}$ values were less than $-6.0 \mathrm{kcal} / \mathrm{mol}$. Among all the test compounds, SET had the best binding energy and $\log \mathrm{P}$ value combination (-8.78 and 0.78 , respectively) and can be found close to the other IBDs in the green box on the plot (Figure 3). Compounds 14a, 15a, 17a, 31a, 32a, 35a, 36a, 38a, 39a, 41a, 43a, 45a and 47a (totally, 13 IBDs) were found to have $\log \mathrm{P}$ values $>3.0$ and binding energy ranging between -7.8 to $-9.12 \mathrm{kcal} / \mathrm{mol}$, while a handful (just 4 ) of the compounds (16a, 19a, 34a and 42a) were found to be very hydrophobic with a $\log \mathrm{P}$ value of $>4.0$. Also, these particular IBDs had $\Delta \mathrm{G}$ values of <-8.0. When the $\log \mathrm{P}$ value ranges between 1.5 and 2.1 , compounds are known to go into the brain tissue by crossing the blood-brain barrier (BBB) and molecules with $\log \mathrm{P}>5$ are used as sublingual drugs. SET, 9a and $13 \mathrm{a}$ have $<1.5 \log \mathrm{P}$ and therefore, these compounds may not significantly cross the BBB. Hence, using this plot as a guideline and by taking stock of the previously reported ADME properties of these compounds (Kumar et al., 2020), we can determine that around 10 of the 47 compounds were capable of high affinity interactions with RdRp and were also not extremely hydrophobic. The ADME and structure-activity relationships of these compounds have been charted out by us in our earlier published work. All the molecules obeyed Lipinski's rule of five and exhibited drug-likeness.

\section{Interactions between RdRp and the docked ligands}

The interactions between $\mathrm{RdRp}$ and the control/test molecules were explored by checking the groups/atoms involved in ligand-receptor binding. The key aminoacids and the 
types of interactions involved in docking of the ten best molecules to the RdRp protein are given in table 2.

Control compounds such as REM, SET, SOF and YAK were found to interact with RdRp through a) hydrogen bonding via some amino acid residues which are common, for example-Y619, D623, R624, D760, N691. Other unique residues were also involved in hydrogen bonding between H-bond donors and acceptors found in both the ligand and receptor. These bonds stabilize the incoming ligand and facilitate its docking to a favourable site. Taking REM as an example, we can see that the multiple interactions of hydrogen bonding + hydrophobic contacts $+\pi-\pi$ interactions were found to be responsible for stabilization of the receptor-ligand complex. Hydrogen bonding of REM via RdRp residues D623 and R6244 (shown as blue arrows in Figure 4A) occurred when one Asp (D623) acted as an H-bond acceptor, while an O-atom of REM bonded with R624, an H-bond donor. A series of other amino acid residues were found to make hydrophobic/van der Waal's contacts/other non-covalent interactions with REM, such as K621, Y619, D452, T680, S682, S681, T687, and N691. Weak intermolecular forces often stabilize interactions by aiding the conformation of chemical compounds and contribute to the overall binding energy. A few $\pi-\pi$ interactions (shown by orange dotted lines) facilitated the interaction of aromatic residues with phenyl/other aromatic groups of the ligand. Similarly, panels B-E show receptor-ligand interactions between four other control drugs and panels E-N reveal the interactions that are most likely to occur between $\mathrm{RdRp}$ and the ten selected IBDs. More $\pi-\pi$ interactions between RdRp-IBDs can be seen and this may be a reason for the better $\Delta \mathrm{G}$ values and increased stability of the receptor-ligand complexes. The interactions for all other compounds (both controls and IBDs) are given in Table 2 and Table S4. Also, the interactions of the ten best IBDs with RdRp residues are shown in Figure 4 and the interactions of the 5 best energy controls are also presented. 
Among the 47 isatin compounds, 45 compounds were found to bind to the palm and the finger regions of RdRp, while 2 IBDs (10a and 12a) failed to bind. Most of the compounds interacted with key residues involved in metal ion $\left(\mathrm{Mg}^{2+}\right)$ binding and stabilization, such as D623 and also docked very close to the critical catalytic residues mentioned earlier (Gao et al., 2020). Therefore, binding of the best IBDs to the palm and finger domains may preclude substrate interaction with RdRp and decrease viral RNA levels. The binding of REM is compared to the docking site of 28a (Figure 5). Almost all the compounds explored in the present study were found to bind to the RNA channel of RdRp. The overlap of the chemical compounds (control as well as the ten best IBDs) is presented in Figure S1.

\section{Molecular Dynamics Simulation of Native and Ligand Bound Complexes of RdR polymerase}

MD studies on the native RdRp protein yields basic understanding of the structural behavior and the stability of the protein during a time period of 100 ns. In molecular dynamics simulation studies, Root Mean Square Deviation (RMSD) and Root Mean Square Fluctuation (RMSF) are good indicators for analysis of protein stability and flexibility. In native RdRp protein dynamics, the average backbone RMSD values were found to be in the range of $0.5 \mathrm{~nm}$ and the data reveals that the structural stability of the protein was maintained throughout 100 ns time period (Figure 6). Also, RMSF profiles show that residues in the Nterminal (first 100 residues) and C-terminal domain (last 30 residues) fluctuated greatly in the range of 0.2-0.9 and 0.2-0.6 nm, respectively, due to its relaxation and flexibility (Figure 7). Overall, the native RdRp protein shows higher structural stability during 100 ns time period, therefore, the RMSD and RMSF values obtained with the native protein (only RdRp) can be considered as reference values with which, the data for ligand-bound RdRp complexes can be 
compared to support the binding energies obtained through molecular docking and also to ensure the reliability of the simulation study.

Here, the structural stability and potential interactions of RdRp-REM complex obtained from the MDS study was consider to compare with the ligand-bound (Compound16, 24, 28, 38 and 40) complexes of RdRp protein. In RdRp-REM complex, the average RMSD value was in the range of $0.3-0.5 \mathrm{~nm}$ and RMS fluctuation found $0.2-0.9 \mathrm{~nm}$, almost identical to the native (un-liganded) RdRp protein. But, in the initial $50 \mathrm{~ns}$ time periods, the RMSD values found to be under $0.3-0.45 \mathrm{~nm}$, after which, deviation increased up to $0.5 \mathrm{~nm}$ due to REM's conformational changes and loss of h-bond interactions with Lys621, Arg670, and Arg671 in the protein. Also, it is noteworthy to analyze the potential H-bond interactions of REM in the RdRp protein. The H-bond occupancies of the complex showed that the potential residues Tyr455, Lys545, Arg553, Arg555, Lys621, Asp623, Arg624, Asp760 Asp761, and Glu811 are found to interact with REM during 100 ns time period and the corresponding occupancy values (more than 20\%) are displayed in the Figure 8a.

The binding free energy of the RdRp-REM complex was calculated by the MMPBSA method. The electrostatic interaction energy, van der Waals energy, SASA and total energy of the complex was found to be $-118.6,-106.24,-12.6$, and $-79.7 \mathrm{KJ} / \mathrm{mol}$, respectively (Table. 3). The potential H-bond forming residues contributed to the binding free energy of RdRpREM complex is shown in the Figure 9a. The residues Tyr455, Lys545, Arg553, Arg555, Lys621, Asp623, Arg624, Asp760 Asp761 and Glu811 were found to involve in binding free energies and in attainment of energetically favourable/stable conformers of RdRp-isatin complexes. And, residues Arg553, and Asp624 showed more than $-5 \mathrm{kcal} / \mathrm{mol}$ values and remaining all mentioned residues yielded lower values in the complex.

The MDS studies of RdRp bound complexes (RdRp-Compound-16, 24, 28, 38, and 40) reveals that all the complexes were highly stable compared to the native RdRp and RdRp- 
REM complex during 100 ns time periods. The RMSD plot clearly shows that the average deviation of the complexes was found between 0.3 and $-0.45 \mathrm{~nm}$ while the RMSF plot shows the residual fluctuation values of $\mathrm{N}$ - and C-terminal domains to occur in the range of $0.7 \mathrm{~nm}$ (Figure 6 and 7). Moreover, all the five complexes were found to be slightly deviated in the initial $50 \mathrm{~ns}$ time periods (equilibration time), after which, the complexes stabilized and maintains the structural integrity. Compared to the RMSD and RMSF values of RdRp-REM complex, all the five complexes were found to be stable and less fluctuations depicted the inhibitory potential of the compounds. The potential h-bond interactions and their corresponding occupancy values during 100 ns time period were shown in Figure 8b-f. All the five compounds were found to have high H-bond occupancy values compared with Remdesvir complex; hence, these compounds produced higher stability and may involve in the better inhibitory activity against the RdRp protein. Significantly, the residues Asp623 and Arg624 maximally forms h-bond interaction with compounds during $100 \mathrm{~ns}$ time periods and predicted to be important for the stable complex formation. The binding free energy of the complexes calculated by the MMPBSA is shown in Table 3. All the five complexes electrostatic interaction energy, van der waals energy, SASA and total energies were lower than the RdRp-remdesvir complex, signifying its role on the protein structure. RdRpCompound-28 showed higher electrostatic interaction energy, van der waals energy, SASA and total energies values of $-147.6,-183.5,-16.3$ and -176 , respectively (Table. 3). Moreover, the residues which were involved in proffering the binding free energy of the complexes are shown in Figure 9b-f, which suggests that these residues are important for the complex formation and could play a vital role in the inhibitory activity. Collectively, all the five compounds showed better stability and binding free energy compared with reported REM complex and therefore, these compounds may produce better inhibitory activity against the RdRp protein than other currently available standard drug molecules acting on RdRP. 


\section{DFT studies}

Calculating the charge transfer reactions of the molecules (donor and acceptor) is an important aspect/parameter in the investigation of chemical reaction mechanisms. Here, the frontier molecular orbitals (FMOs) such as HOMO (electron donor) and LUMO (electron acceptor) energy compounds were calculated for the top-scored compounds. HOMO-LUMO components of the molecules are involved in molecular interactions with the RdRp protein receptor residues; in this context, the highest molecular orbitals were predicted to decipher the mode of complex formation as well as the reaction of the receptor and isatins. In compounds-16, 24 and 28, HOMO regions localized in the oxindole group of five-member nitrogen containing ring, whereas LUMO regions localized in the thiourea and urea group of the compounds (Figure 10a-c). In compound-38, HOMO region was located in the spriochromene group and the LUMO region localized in the oxindole group of six-member benzene ring (Figure. 10d). Significantly, compound-40 has only HOMO region spreads over the central ring (oxindole group) and the side chain of the molecule (Figure 10f). HOMOLUMO energy values and the energy gap of the five compounds are shown in Table. 4. The HOMO-LUMO energy gap ( $\left.\mathrm{E}_{\mathrm{HOMO}}-\mathrm{E}_{\mathrm{LUMO}}\right)$ signifies the stability and chemical reactivity of molecules. Lowering the energy gap can influence the charge transfer reaction and thereby, determine the biological activity of the molecules. Overall, all the five molecules with higher HOMO values in the structures, could have participated in the nucleophilic reaction and the lower energy gap values of these compounds are expected to yield better inhibitory activities.

\section{ADME profiling:}

ADME predictions show that the compound A28 possesses safer ADMET parameters than other compounds (A16, A24, A 38 and A40). This compound is anticipated to have druggable properties due to favourable metabolic parameters. Therefore, exploring the 
inhibitory activities of the small molecule derivatives can facilitate to expand the repertoire of promising inhibitors that can mitigate the infection and the transmission of the virus (Table S5).

\section{Conclusion}

Based on the in-silico data obtained (binding energies, $\log \mathrm{P} / \mathrm{S}+\log \mathrm{P}$ and $\mathrm{S}+\log \mathrm{D}$ ), 10 of the total 47 IBDs which were explored in this work can be considered as promising drug molecules for in vitro investigations. The docking data, dynamics and DFT studies have evidenced that some of these compounds (specifically, compounds 16, 24, 28, 38 and 40) could be potentially novel therapeutics against RdRp of SARS-CoV-2. Some of the IBDs synthesized by us were found to have better $\Delta \mathrm{G}$ and $\log \mathrm{P}$ values when compared to most of the standard COVID-19 drugs, including REM. The molecular dynamics simulation studies revealed that $\mathrm{RdRp}$ complexes of all the five compounds were found more stable compared with REM complex. While REM is known to terminate RNA synthesis by forming an RMP intermediate, the other standard drugs also were remarkably much less favoured (energetically) than the isatin derivatives reported in this work. Also, total binding free energies and residues decomposition energies of all the five complexes of $R \mathrm{dRp}$ were found to be higher when compared with RdRp-REM complexes. DFT studies showed that the identified five compounds have higher HOMO values than LUMO values, and hence, these molecules may be involved in nucleophilic reaction of the drug with residues within the RdRp binding pocket. We deem this to be the prospective inhibitory mechanism of the IBDs studied in this work. Among the best RdRp-IBD complexes, ADME profiling revealed that A28 is a possible lead compound which can be explored further for anti-RdRp activity in vitro. The SARS-CoV-2 RdRp is the critical enzyme for viral propagation. Recently the proposed functional form of Holo-RdRp (RdRp+NSP8+NSP7+RNA) in complex with NSP13 helicase depicts the replication/transcription machinery which is pivotal for the 
efficient enzymatic activity (Chen et al., 2020). This key enzyme has utmost functional significance and is a prime target for developing therapeutics. Therefore inhibiting this enzyme is the most viable approach to treat COVID-19 because we can knock down the capacity of the virus to propagate. On exploring the binding as well as the dynamic action of Isatin-based derivatives against $R d R p$, it is evident that most of the compounds show better binding affinity as well as overall dynamical stability. We plan to assess the in vitro RdRpinhibitory activity of the best IBDs identified in this work in the near future.

\section{Declaration of interests}

The authors declare that they do not have any competing interests to disclose. 


\section{Acknowledgements}

JJ sincerely thanks the UGC-RGNF, New Delhi, India (Ref. No: RGNF-2015-17- SCTAM17403) for financial assistance. We thank Department of Chemistry, Bishop Heber College, Tiruchirappalli, India for support. JJ greatly acknowledges Department of Science and Technology (F.No.EMR/2016/000498 dated: 26.09.2016), Indian Council of Medical Research (ICMR) (No.BIC/12(07)/2015), Board of Research in Nuclear Sciences (BRNS) (35/14/02/2018-BRNS/35009) and DST Indo-Taiwan (GITA/DST/TWN/P-86/2019) for providing financial assistance through Major research projects. The authors thank DST-FIST (SR/FST/LSI-667/2016), New Delhi for providing infrastructure facilities. Authors sincerely acknowledge the Department of Science and Technology, New Delhi for the financial support in general and infrastructure facilities sponsored under PURSE 2nd Phase programme (Order No. SR/PURSE Phase 2/38 (G) dated: 21.02.2017) and MHRD-RUSA 2.0,(F.24-51/2014-U, Policy (TNMultiGen), Dept. of Edn. Govt. of India, Dt.09.10.2018.

RM acknowledges UGC-RGNF, New Delhi, India (Ref. No: RGNF-2015-17-SCTAM17403) for financial assistance. VD thanks the management, Bishop Heber College for financial support. 


\section{References}

Ahmad, M., Dwivedy, A., Mariadasse, R., Tiwari, S., Kar, D., Jeyakanthan, J., and Biswal, B.K. (2020). Prediction of Small Molecule Inhibitors Targeting the Severe Acute Respiratory Syndrome Coronavirus-2 RNA-dependent RNA Polymerase. ACS omega 5, 18356-18366.

Buonaguro, L., Tagliamonte, M., Tornesello, M.L., and Buonaguro, F.M. (2020). SARSCoV-2 RNA polymerase as target for antiviral therapy. Journal of Translational Medicine 18, $1-8$.

Chen, J., Malone, B., Llewellyn, E., Grasso, M., Shelton, P.M., Olinares, P.D.B., Maruthi, K., Eng, E.T., Vatandaslar, H., and Chait, B.T. (2020). Structural basis for helicase-polymerase coupling in the SARS-CoV-2 replication-transcription complex. Cell 182, 1560-1573. e1513.

Choubey, S.K., Nachiappan, M., Richard, M., Chitra, J.P., and Jeyakanthan, J. (2020). Structural and functional insights of STAT2-NS5 interaction for the identification of NS5 antagonist-An approach for restoring interferon signaling. Computational Biology and Chemistry 88, 107332.

Choy, K.-T., Wong, A.Y.-L., Kaewpreedee, P., Sia, S.-F., Chen, D., Hui, K.P.Y., Chu, D.K.W., Chan, M.C.W., Cheung, P.P.-H., and Huang, X. (2020). Remdesivir, lopinavir, emetine, and homoharringtonine inhibit SARS-CoV-2 replication in vitro. Antiviral research, 104786.

Cohn, M. (1953). A study of oxidative phosphorylation with O18-labeled inorganic phosphate. Journal of Biological Chemistry 201, 735-750.

Dileepan, A.B., Prakash, T.D., Kumar, A.G., Rajam, P.S., Dhayabaran, V.V., and Rajaram, R. (2018). Isatin based macrocyclic Schiff base ligands as novel candidates for antimicrobial and antioxidant drug design: In vitro DNA binding and biological studies. Journal of Photochemistry and Photobiology B: Biology 183, 191-200.

Eggadi, V., Kulandaivelu, U., Sharvanabhava, B., and Jupally, V.R. (2013). Screening of the anticonvulsant activity of some isatin derivatives in experimental seizure models and its effect on brain GABA levels in mice. Am J Pharmacol Sci 1, 42-46.

Elfiky, A.A. (2020). Ribavirin, Remdesivir, Sofosbuvir, Galidesivir, and Tenofovir against SARS-CoV-2 RNA dependent RNA polymerase (RdRp): A molecular docking study. Life sciences, 117592.

Eros, D., Kovesdi, I., Orfi, L., Takacs-Novak, K., Acsády, G., and Kéri, G. (2002). Reliability of $\log \mathrm{P}$ predictions based on calculated molecular descriptors: a critical review. Current medicinal chemistry 9, 1819-1829.

Gao, Y., Yan, L., Huang, Y., Liu, F., Zhao, Y., Cao, L., Wang, T., Sun, Q., Ming, Z., and Zhang, L. (2020). Structure of the RNA-dependent RNA polymerase from COVID-19 virus. Science $368,779-782$.

Goodsell, D.S., Morris, G.M., and Olson, A.J. (1996). Automated docking of flexible ligands: applications of AutoDock. Journal of molecular recognition 9, 1-5.

Gordon, C.J., Tchesnokov, E.P., Feng, J.Y., Porter, D.P., and Götte, M. (2020a). The antiviral compound remdesivir potently inhibits RNA-dependent RNA polymerase from Middle East respiratory syndrome coronavirus. Journal of Biological Chemistry 295, 4773-4779. 
Gordon, C.J., Tchesnokov, E.P., Woolner, E., Perry, J.K., Feng, J.Y., Porter, D.P., and Götte, M. (2020b). Remdesivir is a direct-acting antiviral that inhibits RNA-dependent RNA polymerase from severe acute respiratory syndrome coronavirus 2 with high potency. Journal of Biological Chemistry 295, 6785-6797.

Kumar M, R., and Alagumuthu, M. (2018). Synthesis and Molecular Drug Efficacy of Indoline-based Dihydroxy-thiocarbamides: Inflammation Regulatory Property Unveiled over COX-2 Inhibition, Molecular Docking, and Cytotoxicity Prospects. Journal of Heterocyclic Chemistry 55, 1658-1668.

Kumar, M.R., Dhayabaran, V.V., Sudhapriya, N., Manikandan, A., Gideon, D.A., and Annapoorani, S. (2020). p-TSA. H2O mediated one-pot, multi-component synthesis of isatin derived imidazoles as dual-purpose drugs against inflammation and cancer. Bioorganic Chemistry, 104046.

Kumar, M.R., Manikandan, A., Sivakumar, A., and Dhayabaran, V.V. (2018). An ecofriendly catalytic system for multicomponent, one-pot synthesis of novel spiro-chromeno indoline-triones and their anti-prostate cancer potentials evaluated via alkaline phosphatase inhibition mechanism. Bioorganic chemistry 81, 44-54.

Langeswaran, K., Jeyaraman, J., Mariadasse, R., and Soorangkattan, S. (2018). Insights from the Molecular modeling, docking analysis of illicit drugs and Bomb Compounds with Honey Bee Odorant Binding Proteins (OBPs). Bioinformation 14, 219.

Li, Y.C., Bai, W.Z., and Hashikawa, T. (2020). The neuroinvasive potential of SARS-CoV2 may play a role in the respiratory failure of COVID-19 patients. Journal of medical virology 92, 552-555.

Liu, C., Zhou, Q., Li, Y., Garner, L.V., Watkins, S.P., Carter, L.J., Smoot, J., Gregg, A.C., Daniels, A.D., and Jervey, S. (2020). Research and development on therapeutic agents and vaccines for COVID-19 and related human coronavirus diseases (ACS Publications).

Mariadasse, R., Choubey, S.K., and Jeyakanthan, J. (2019). Insights into Exogenous Tryptophan-Mediated Allosteric Communication and Helical Transition of TRP Protein for Transcription Regulation. Journal of Chemical Information and Modeling 60, 175-191.

Medvedev, A., Buneeva, O., and Glover, V. (2007). Biological targets for isatin and its analogues: implications for therapy. Biologics: targets \& therapy 1,151 .

Murugan, N.A., Pandian, C.J., and Jeyakanthan, J. (2020). Computational investigation on Andrographis paniculata phytochemicals to evaluate their potency against SARS-CoV-2 in comparison to known antiviral compounds in drug trials. Journal of Biomolecular Structure and Dynamics, 1-12.

Pandeya, S.N., Smitha, S., Jyoti, M., and Sridhar, S.K. (2005). Biological activities of isatin and its derivatives. Acta Pharm 55, 27-46.

Rajesh Kumar, M., Alagumuthu, M., and Violet Dhayabaran, V. (2018). N-substituted hydroxynaphthalene imino-oxindole derivatives as new class of PI 3-kinase inhibitor and breast cancer drug: Molecular validation and structure-activity relationship studies. Chemical biology \& drug design 91, 277-284. 
Visualiser, D.S. 2.5. 1, Accelrys Software Inc. Discovery Studio Modeling Environment, Release 2.

Wu, A., Peng, Y., Huang, B., Ding, X., Wang, X., Niu, P., Meng, J., Zhu, Z., Zhang, Z., and Wang, J. (2020). Genome composition and divergence of the novel coronavirus (2019-nCoV) originating in China. Cell host \& microbe.

Yang, X., Yu, Y., Xu, J., Shu, H., Liu, H., Wu, Y., Zhang, L., Yu, Z., Fang, M., and Yu, T. (2020). Clinical course and outcomes of critically ill patients with SARS-CoV-2 pneumonia in Wuhan, China: a single-centered, retrospective, observational study. The Lancet Respiratory Medicine.

Yin, W., Mao, C., Luan, X., Shen, D.-D., Shen, Q., Su, H., Wang, X., Zhou, F., Zhao, W., and Gao, M. (2020). Structural basis for inhibition of the RNA-dependent RNA polymerase from SARS-CoV-2 by remdesivir. Science. 
Tables and Figures:

Table 1. Docking results for 10 selected IBDs and a few RdRp inhibitors (positive controls)

\begin{tabular}{|c|c|c|c|c|c|c|c|c|c|}
\hline $\begin{array}{c}\text { (Ligand) } \\
\text { Imidazole } \\
\text { derivatives }\end{array}$ & $\begin{array}{c}\text { Binding } \\
\text { energy } \\
\text { (kcal/mol) }\end{array}$ & $\begin{array}{l}\text { Ligand } \\
\text { efficiency }\end{array}$ & $\begin{array}{c}K_{\mathbf{i}} \\
(\boldsymbol{\mu M})\end{array}$ & IE & Vdw_hb_DE & ESE & TIE & TE & $\mathbf{U E}$ \\
\hline $13 \mathrm{a}$ & -8.86 & -0.28 & 0.317 & -10.36 & -10.42 & 0.06 & -1.32 & 1.4 & -1.32 \\
\hline $14 \mathrm{a}$ & -8.81 & -0.33 & 0.348 & -10.0 & -9.8 & -0.2 & -0.29 & 1.19 & -0.29 \\
\hline $16 \mathrm{a}$ & -9.18 & -0.28 & 0.188 & -10.67 & -10.45 & -0.21 & -1.75 & 1.49 & -1.75 \\
\hline $18 \mathrm{a}$ & -8.61 & -0.28 & 0.492 & -10.4 & -9.16 & -1.23 & -0.94 & 1.79 & -0.94 \\
\hline $19 \mathrm{a}$ & -8.73 & -0.26 & 0.397 & -10.52 & -10.27 & -0.25 & -2.14 & 1.79 & -2.14 \\
\hline $23 a$ & -8.84 & -0.29 & 0.332 & -10.93 & -9.55 & -1.38 & -1.76 & 2.09 & -1.76 \\
\hline $24 \mathrm{a}$ & -8.91 & -0.25 & 0.294 & -11.0 & -10.9 & -0.1 & -1.78 & 2.09 & -1.78 \\
\hline $28 \mathrm{a}$ & -9.54 & -0.27 & 0.102 & -11.03 & -10.34 & -0.69 & -1.39 & 1.49 & -1.39 \\
\hline $38 \mathrm{a}$ & -9.12 & -0.28 & 0.206 & -9.12 & -8.78 & -0.34 & 0.0 & 0.0 & 0.0 \\
\hline $40 \mathrm{a}$ & -9.38 & -0.27 & 0.133 & -9.68 & -8.45 & -1.23 & -0.07 & 0.3 & -0.07 \\
\hline \multicolumn{10}{|c|}{ CONTROL DOCKING } \\
\hline REM & -6.55 & -0.16 & 15.91 & -11.62 & -11.07 & -0.55 & -3.92 & 5.07 & -3.92 \\
\hline SET & -8.78 & -0.23 & 0.369 & -10.57 & -10.04 & -0.53 & -2.40 & 1.79 & -2.40 \\
\hline SOF & -6.78 & -0.19 & 10.75 & -10.36 & -9.64 & -0.72 & -2.25 & 3.58 & -2.25 \\
\hline YAK & -8.85 & -0.25 & 0.325 & -10.34 & -10.20 & -0.14 & -1.11 & 1.49 & -1.11 \\
\hline
\end{tabular}

$K_{\mathrm{i}}(\mu \mathrm{M})$ - Inhibition Constant; IE - Internal Energy; Vdw_hb_DE - Vdw_HB_Desolv Energy; ESE - ElectroStatic Energy; TIE Total Internal Energy; TE - Torsional Energy; UE - Unbound Energy 
Table 2. Molecular interactions between SARS-CoV-2 RdRp and control/test drugs:

\begin{tabular}{|c|c|c|c|}
\hline \multicolumn{4}{|c|}{ Best ten IBDs } \\
\hline \multirow[t]{2}{*}{ IBDs } & \multicolumn{3}{|c|}{ Interactions } \\
\hline & Hydrogen Bond & Van der Waal's/other contacts & $\pi-\pi$ interactions \\
\hline $13 \mathrm{a}$ & D623,T680,N691 & K621,R555,A554,Y455,T556,D452,S682 & D623,R553 \\
\hline $14 \mathrm{a}$ & $\mathrm{D} 452$ & Y455,K621 & $\mathrm{R} 624,553$ \\
\hline $16 \mathrm{a}$ & D623 & $\begin{array}{c}\text { Y619,K621,C622,D452,Y455,S682,N691,S681, } \\
\text { T687 }\end{array}$ & R553,R555,R624 \\
\hline $18 \mathrm{a}$ & D623,D760 & $\begin{array}{c}\text { T556,S682,S681,T680,T687,Y455,D452,R624, } \\
\text { Y455,R553,R555 }\end{array}$ & R553 \\
\hline $19 \mathrm{a}$ & D623,D760,C622 & $\begin{array}{l}\text { Y455,R553,Y456,R624,D452,S682,S681,T687, } \\
\text { T556 }\end{array}$ & D623,R553,R624 \\
\hline $23 \mathrm{a}$ & $\begin{array}{l}\text { D452,R553,R555, } \\
\text { D623 }\end{array}$ & D760,Y455,A554 & R553,R624 \\
\hline $24 \mathrm{a}$ & D623,R553,T556 & R555,Y455,N691,T687,P620 & R553,K621,R624 \\
\hline $28 \mathrm{a}$ & R553,D623 & S681,K676,A558,M542,T680,Y456,Y455,K621 & R624,R553,K621 \\
\hline $38 \mathrm{a}$ & R555,R624 & V557,Y455 & R553,R555 \\
\hline $40 \mathrm{a}$ & D623,R553,R555 & Y619,K621,Y455 & R553,K621 \\
\hline \multicolumn{4}{|c|}{ CONTROL COMPOUNDS } \\
\hline \multirow{2}{*}{$\begin{array}{c}\text { RdRp } \\
\text { inhibitors }\end{array}$} & \multicolumn{3}{|c|}{ Interactions } \\
\hline & Hydrogen Bond & $\begin{array}{c}\text { Van der Waal's/other non-polar other } \\
\text { contacts }\end{array}$ & $\pi-\pi$ interactions \\
\hline REM & D623,R624 & K621,Y619,D452,T680,S682,S681,T687,N691 & R553,R624 \\
\hline SET & $\begin{array}{l}\text { Y619,D623,R553, } \\
\text { R624,S682 }\end{array}$ & D618,P620,C622,K621,T680,V557,M542,S681 & R555 \\
\hline SOF & $\begin{array}{c}\text { R624,D452,T556, } \\
\text { R555,D623,D760, } \\
\text { N691 }\end{array}$ & Y455,T680,S682,S681,T687 & R553 \\
\hline YAK & $\begin{array}{l}\text { Y619,D623,D760, } \\
\text { N691 }\end{array}$ & $\begin{array}{c}\text { K621,Y455,A554,D452,T556,R624,Y457,D618, } \\
\text { S682 }\end{array}$ & R624,R553 \\
\hline
\end{tabular}


Table. 3 MMPBSA Binding free energy calculation of RdRp-bound complexes

\begin{tabular}{|c|c|c|c|c|}
\hline Complexes & $\begin{array}{c}\text { Electrostatic } \\
\mathbf{k J} / \mathbf{m o l}\end{array}$ & VDW kJ/mol & $\begin{array}{c}\text { SASA Energy } \\
\mathbf{k J} / \mathbf{m o l}\end{array}$ & $\begin{array}{c}\text { Total Energy } \\
\mathbf{k J} / \mathbf{m o l}\end{array}$ \\
\hline $\begin{array}{c}\text { RdRP- } \\
\text { Remdesivir } \\
\text { (Triphosphate) }\end{array}$ & -118.6 & -106.24 & -12.6 & -79.7 \\
\hline $\begin{array}{c}\text { RdRp- } \\
\text { Compound-16 }\end{array}$ & -126.6 & -126.33 & -3.2 & -149 \\
\hline $\begin{array}{c}\text { RdRp- } \\
\text { Compound-24 }\end{array}$ & -123.6 & -136.23 & -9.3 & -136 \\
\hline $\begin{array}{c}\text { RdRp- } \\
\text { Compound-28 }\end{array}$ & -147.6 & -183.5 & -16.3 & -176 \\
\hline $\begin{array}{c}\text { RdRp- } \\
\text { Compound-38 }\end{array}$ & -139.6 & -179.66 & -19.6 & -156 \\
\hline $\begin{array}{c}\text { RdRp- } \\
\text { Compound-40 }\end{array}$ & -131.66 & -156.96 & -15.3 & -167 \\
\hline
\end{tabular}


Table 4. DFT studies for the top 5 compounds

\begin{tabular}{|c|c|c|c|c|}
\hline S. No & Compounds ID & $\begin{array}{c}\text { HOMO } \\
(\mathbf{e V})\end{array}$ & $\begin{array}{c}\text { LUMO } \\
(\mathbf{e V})\end{array}$ & $\begin{array}{c}\text { EHOMo-ELUMO } \\
(\mathbf{e V})\end{array}$ \\
\hline 1 & 16 & -0.19 & -0.01 & -0.18 \\
\hline 2 & 24 & -0.20 & -0.00 & -0.20 \\
\hline 3 & 28 & -0.19 & -0.03 & -0.16 \\
\hline 4 & 38 & -0.25 & -0.05 & -0.20 \\
\hline 5 & 40 & -0.26 & -0.01 & -0.25 \\
\hline
\end{tabular}




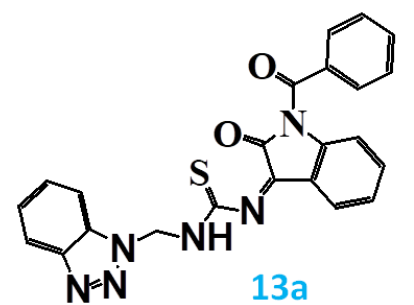

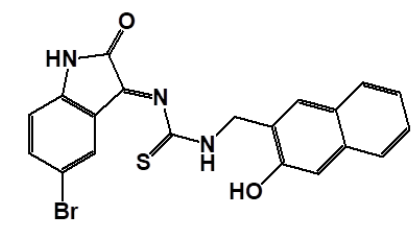

$14 a$

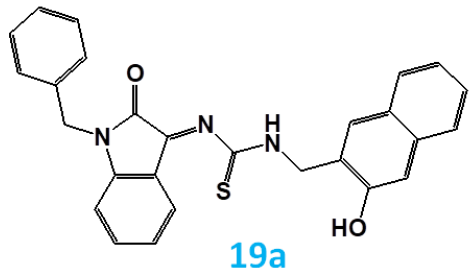

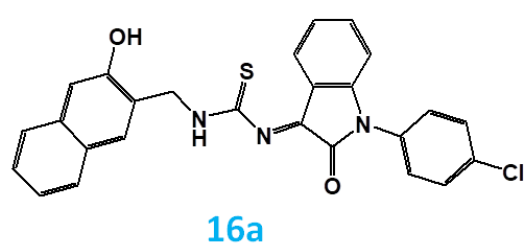
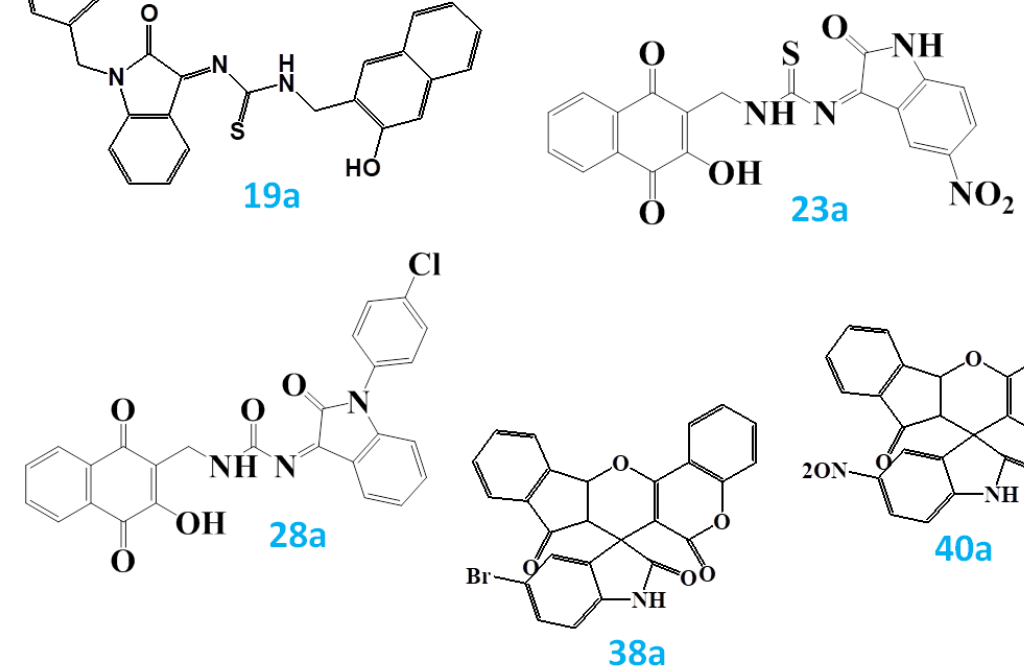

Figure 1. Structure of ten selected IBDs with the best binding energy values 
RdRp binding energy

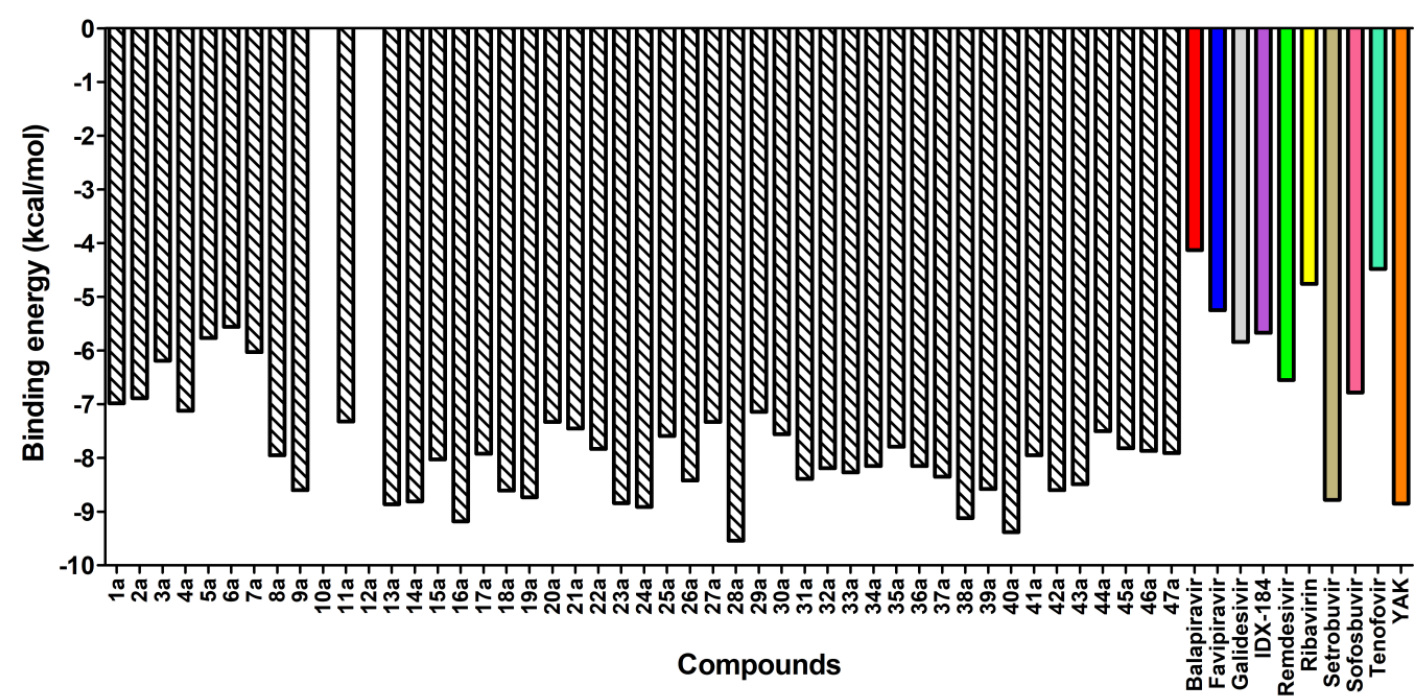

Figure 2. Binding energies of the IBDs and control RdRp inhibitors 


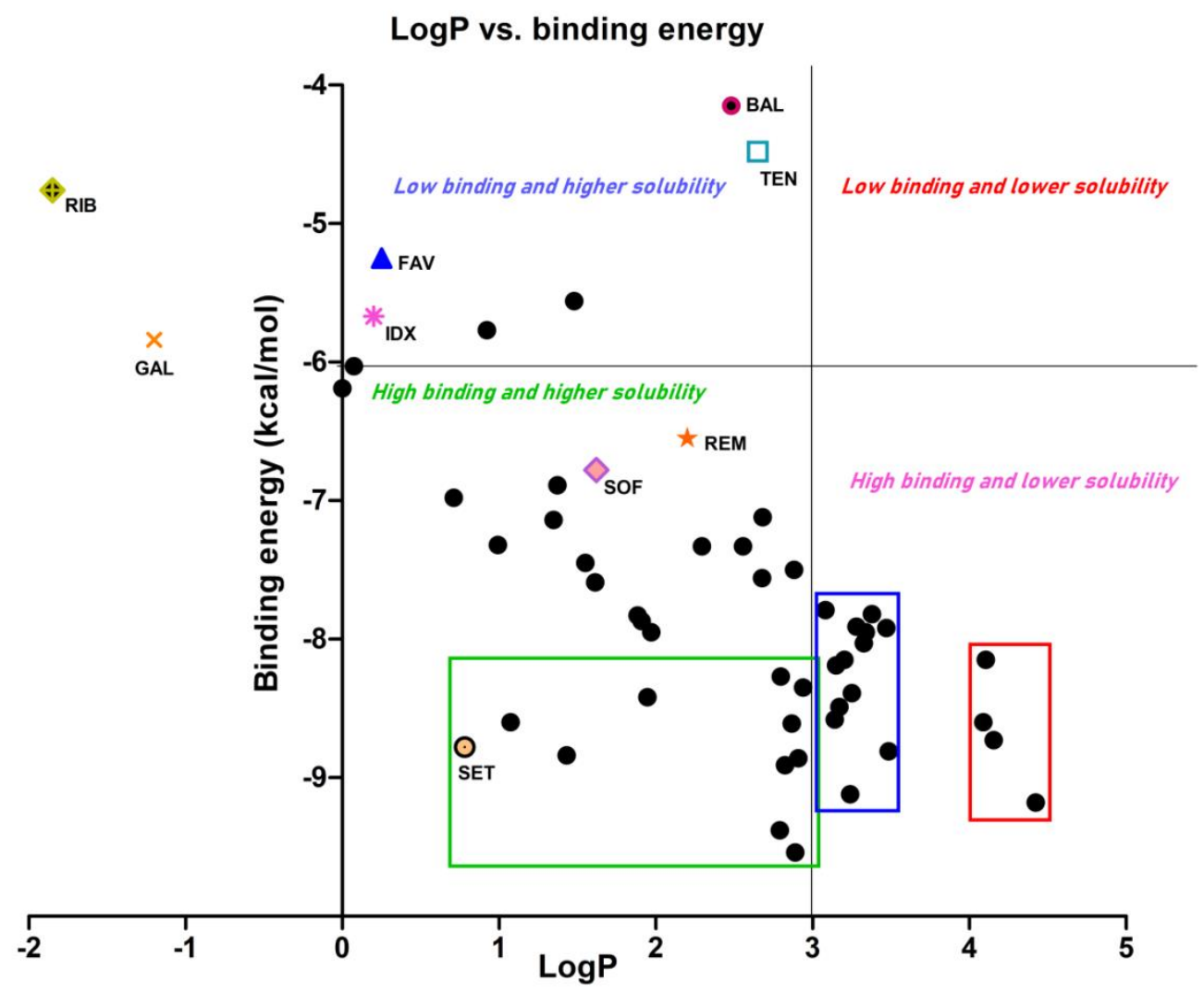

Figure 3. $\operatorname{LogP}$ vs. binding energy plot for control RdRp inhibitors and 47 IBDs: Based on binding energy and $\log \mathrm{P}$ values of the compounds, a plot of energy vs. $\log \mathrm{P}$ was prepared to identify the compounds which can be used for further in vitro explorations. Please refer to the text on Figure 3 for more details. 


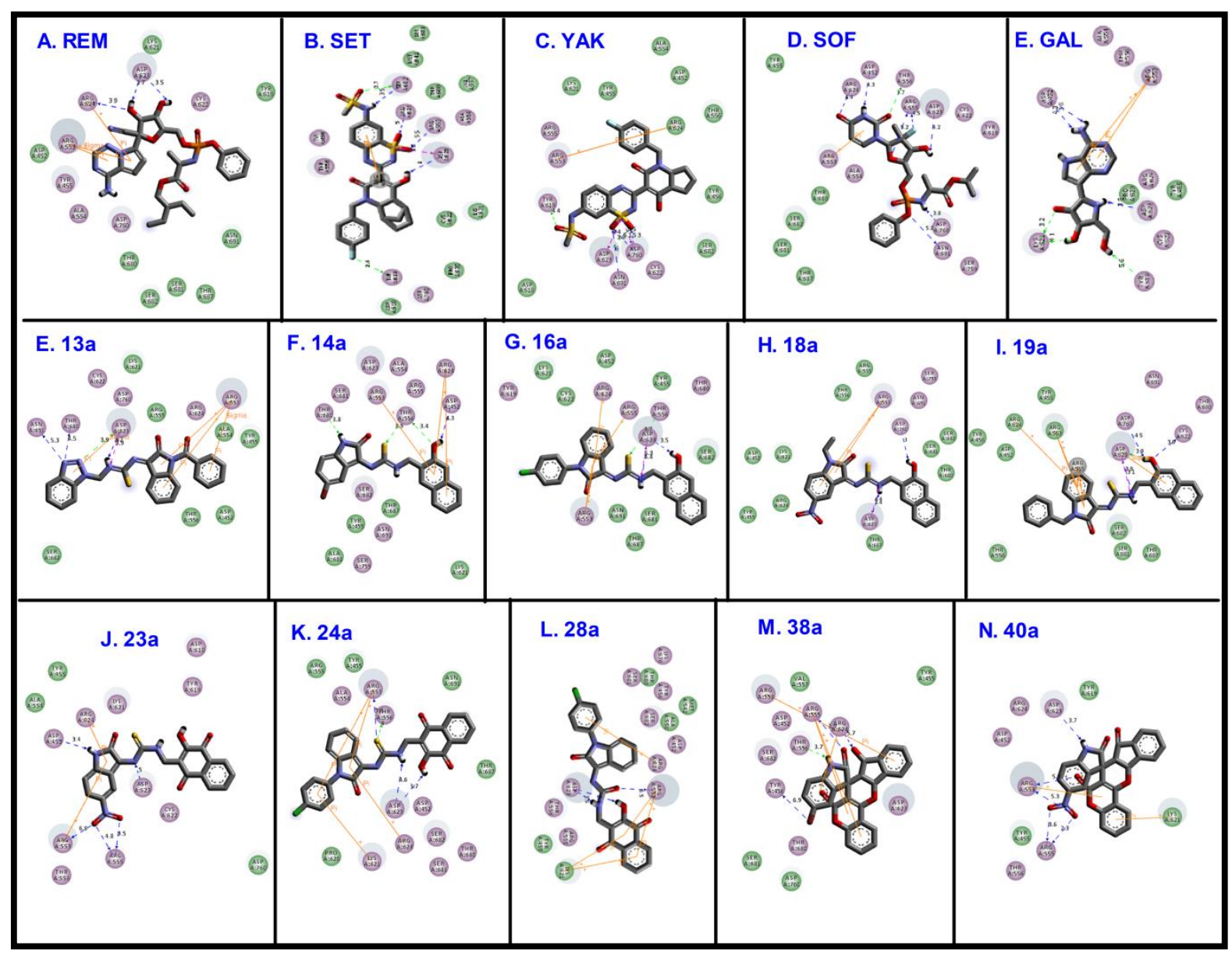

Figure 4. Molecular interactions between SARS-CoV-2 RdRp and control RdRp inhibitors and IBDs: Several types of interactions such as hydrogen bonding, van der Waal's forces, $\pi-\pi$ interactions and hydrophobic interactions may have contributed to the favourable binding energies exhibited in RdRp-IBD interactions. In the Top panel, A-E, the interactions between RdRp and control RdRp inhibitors is shown. At the bottom (panels F-O), the interactions between IBDs and the best hit RdRps are shown. Please see Table 2 and Figure 3 for more details. 

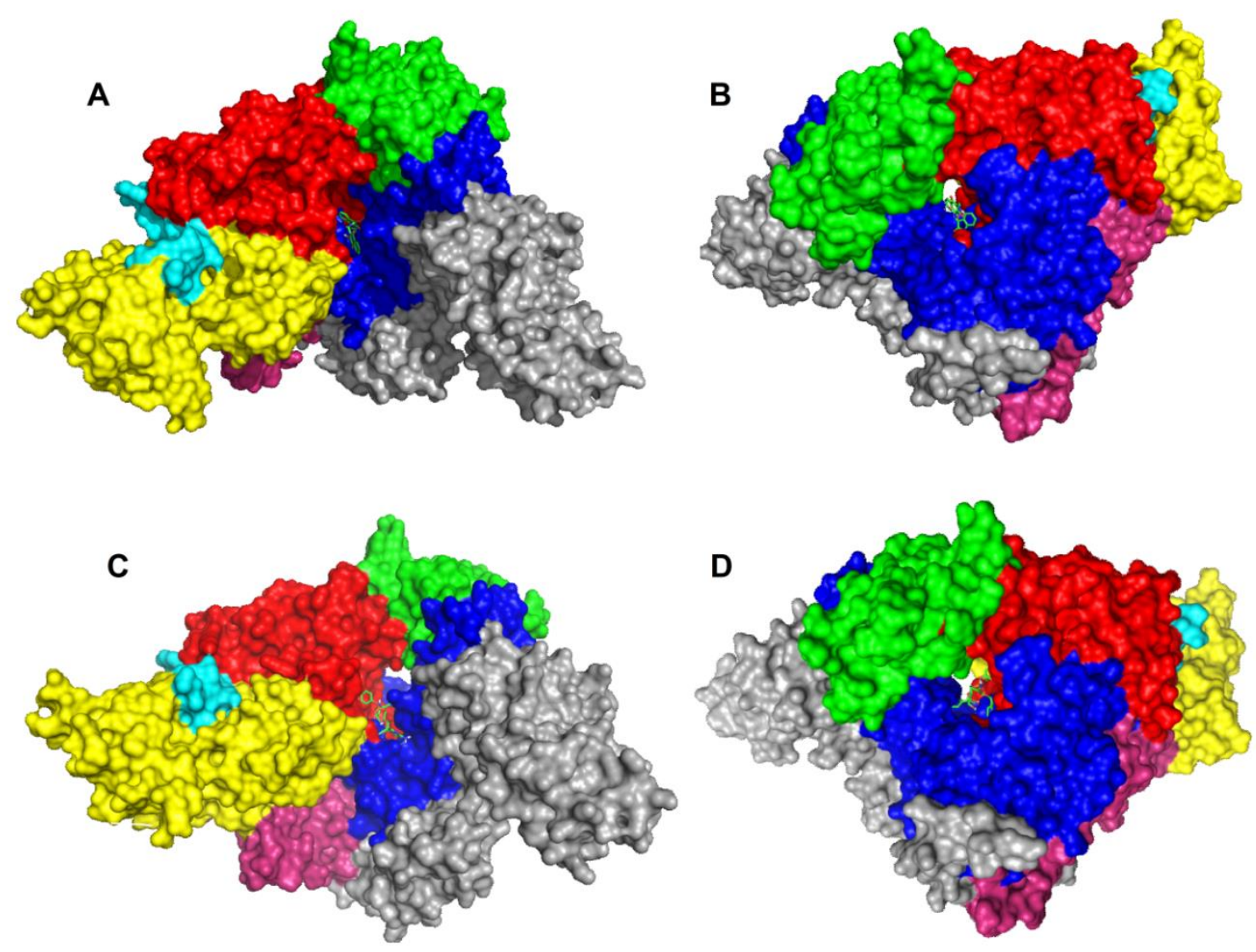

Figure 5. Binding site of 28a and Remdesivir: $\mathbf{A}$ and $\mathbf{B}$ represent REM binding to RdRp the two different sides of the RdRp protein and its central RNA channel is seen as a cavity. In $\mathbf{C}$ and $\mathbf{D}$, binding of 28 a to RdRp is shown from two different sides. The RdRp protein is coloured based on the original rendition of the protein by Gao et. al. (Gao et al., 2020) and the colours are- NiRAN motif 1 and 2 - yellow, $\beta$-clamp - cyan, interface - warm pink, Fingers 1 and 2 - dark blue, Palm 1 and 2 - red, and C-terminal thumb - grey. Almost all the compounds bound to the same place. While REM bound a little outside, close to the edge of the RNA channel, the isatin-based derivative, 28a, was found to dock a little interior to REM in the palm (Cohn) and finger (dark blue) regions. 


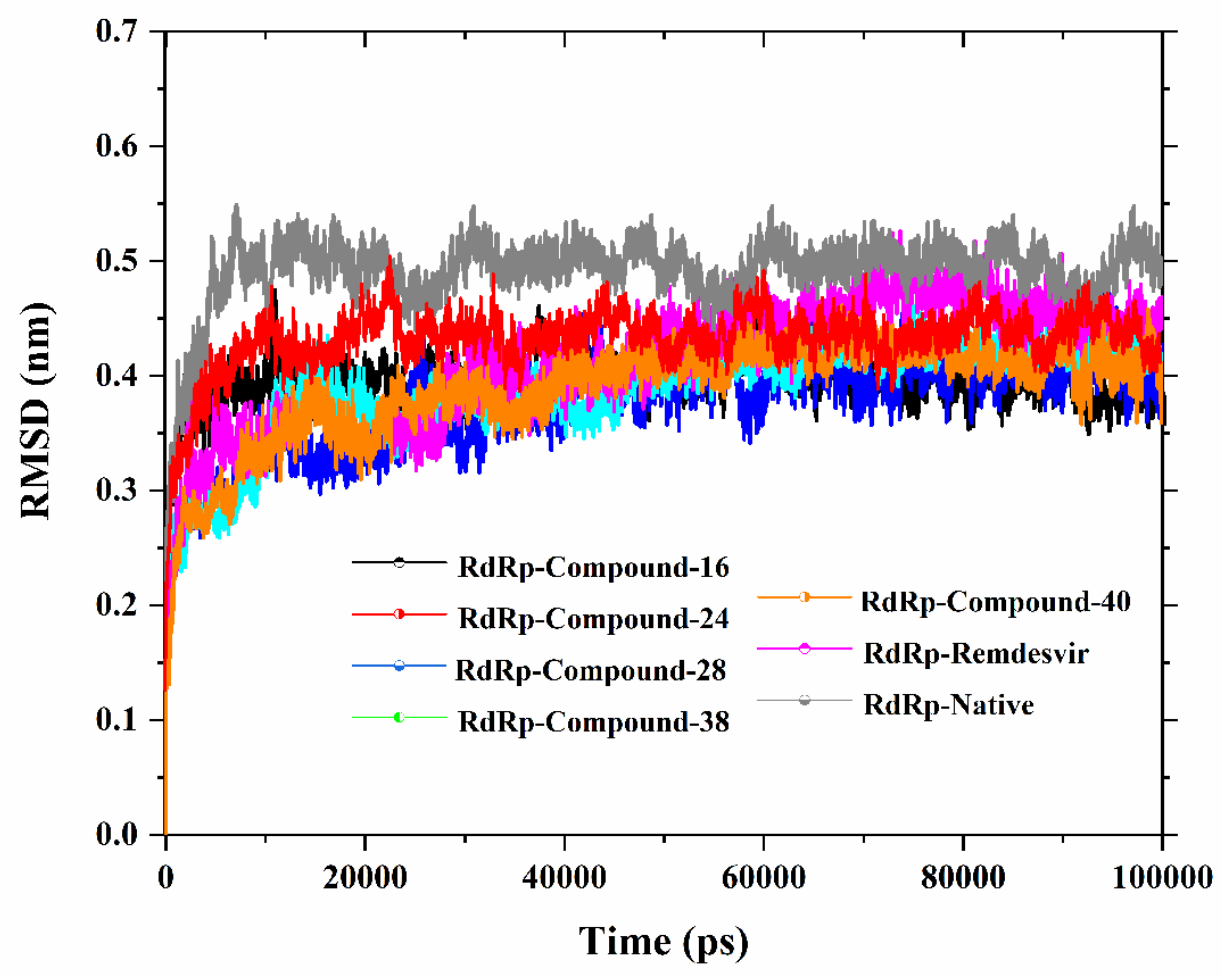

Figure. 6 Comparative RMSD analysis of native and ligand bound complexes of RdRp protein. 


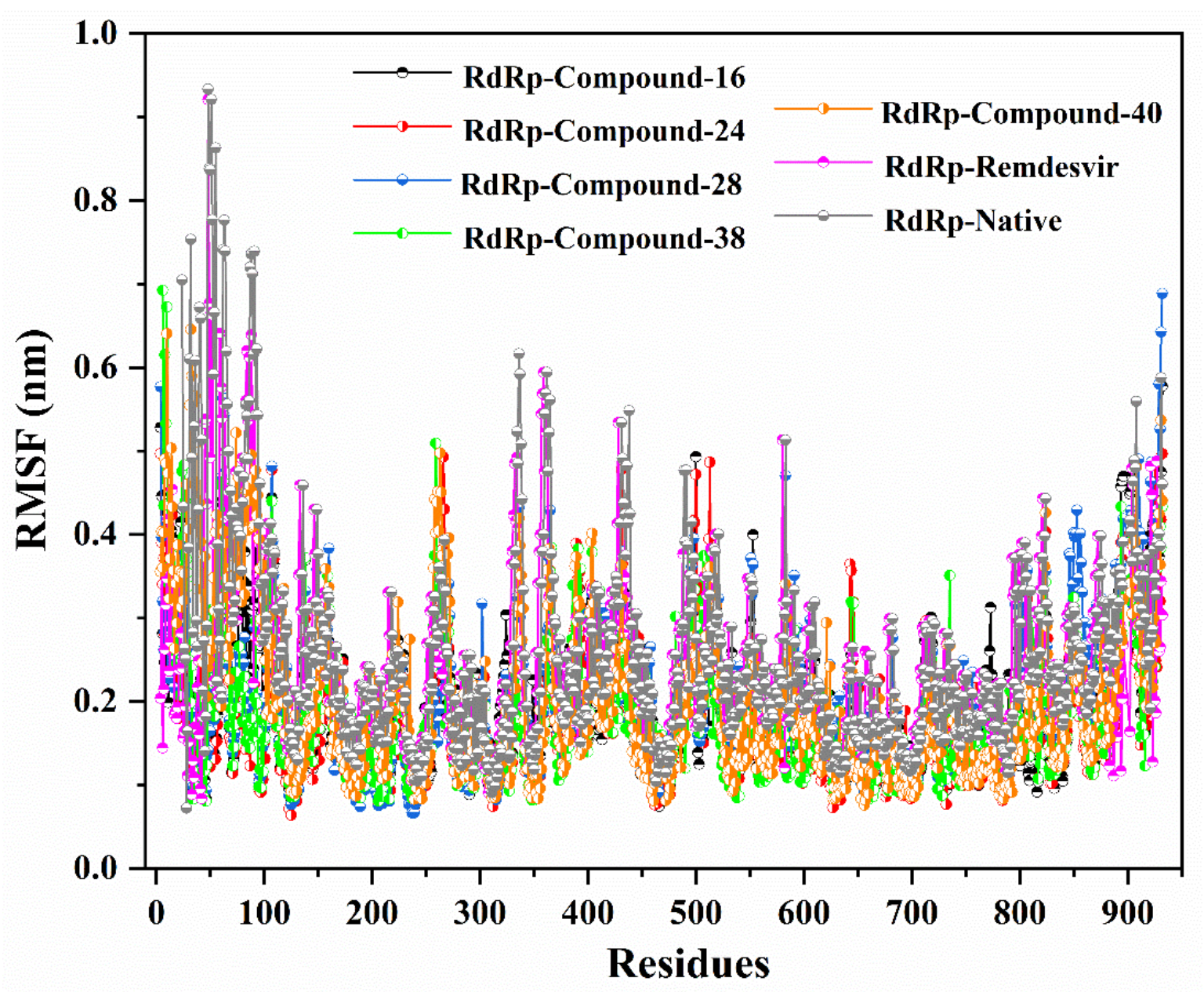

Figure 7. Comparative RMSF analyses of native and ligand bound complexes of RdRp protein 
a)
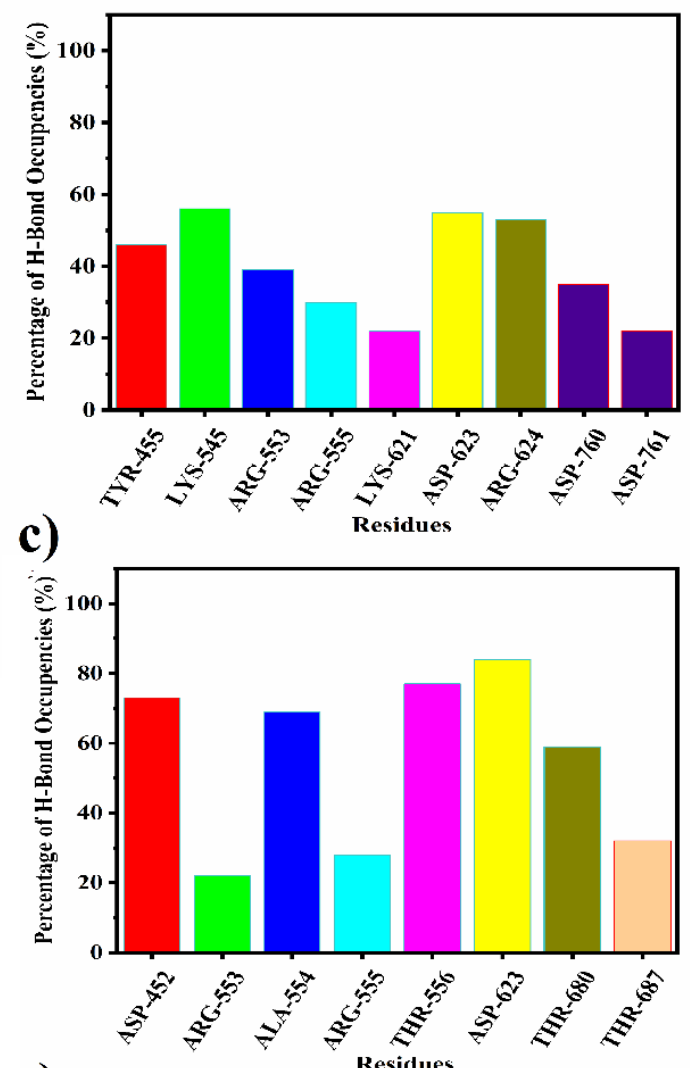

e)

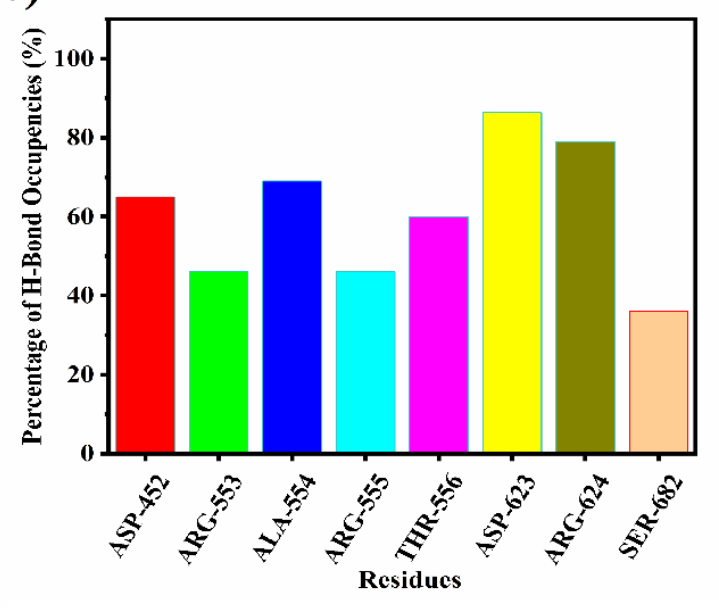

b)
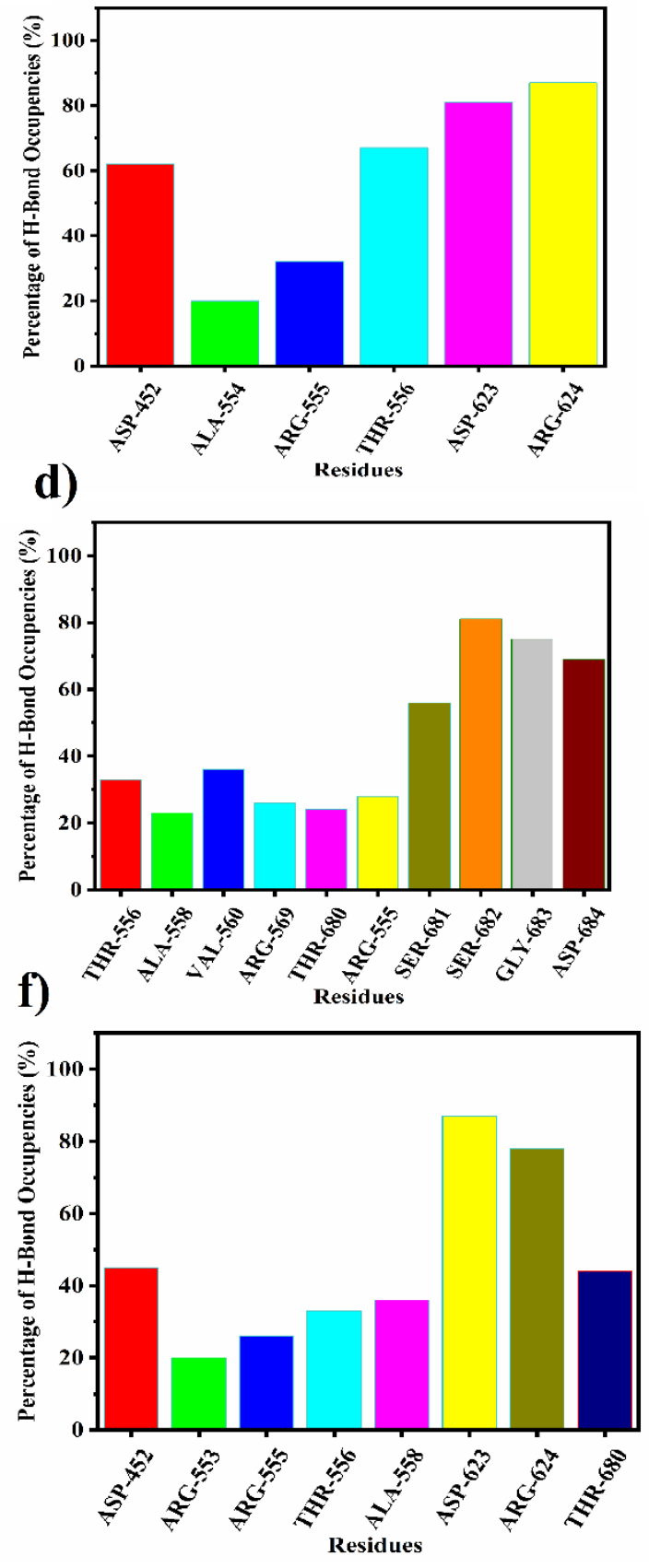

Figure 8. H-bond occupancies of ligand bound complexes of RdRp protein during $100 \mathrm{~ns}$ time period. H-bond occupancy values more than $20 \%$ are displayed here. 

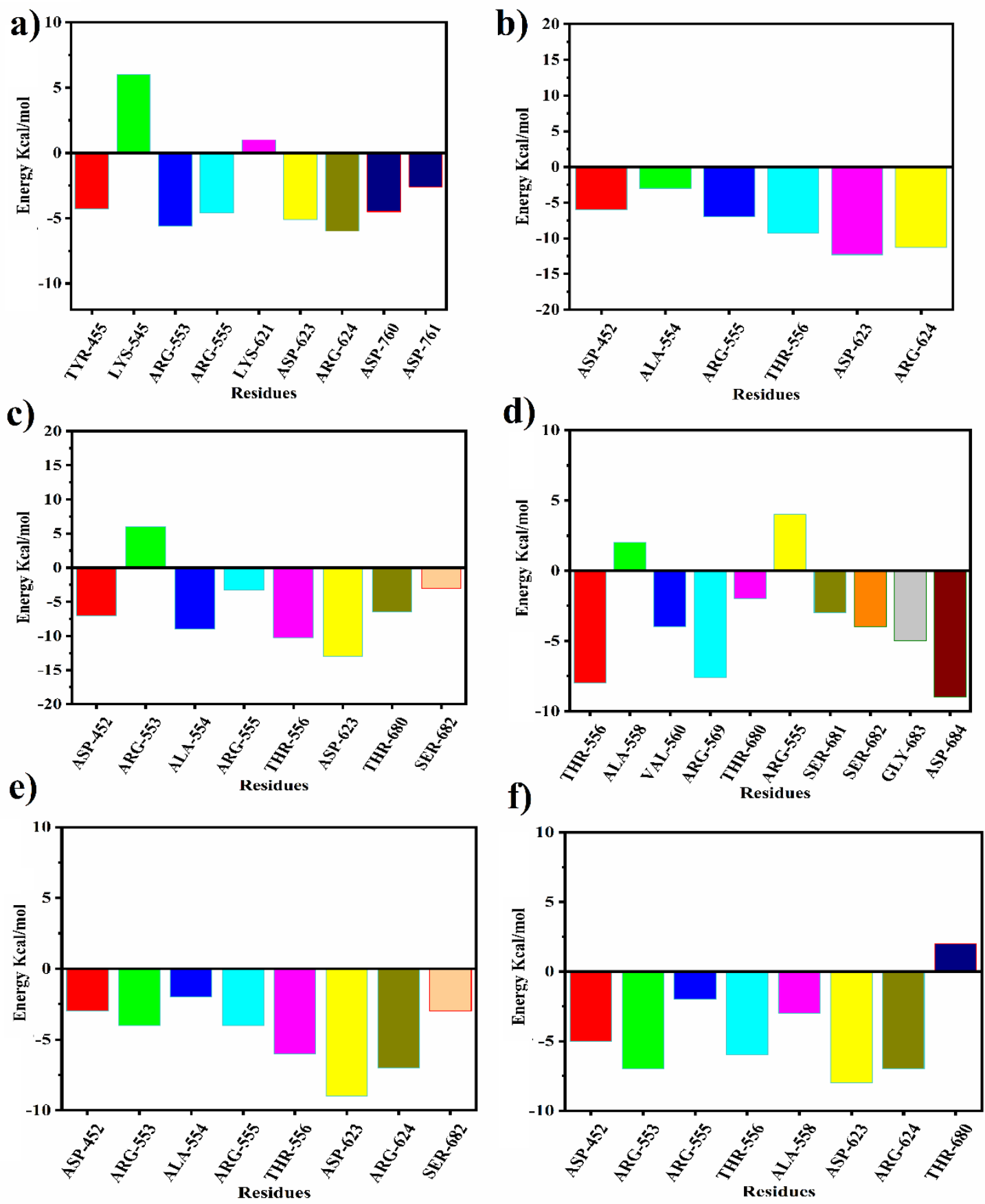

Figure 9. Residual decomposition energies of $R d R p$ protein complexes 
a)

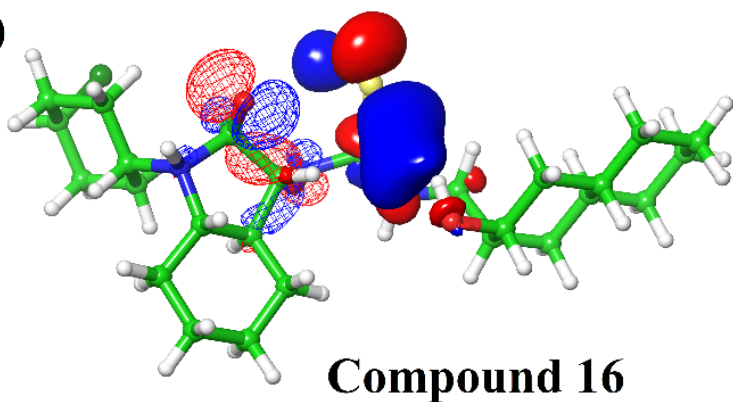

c)

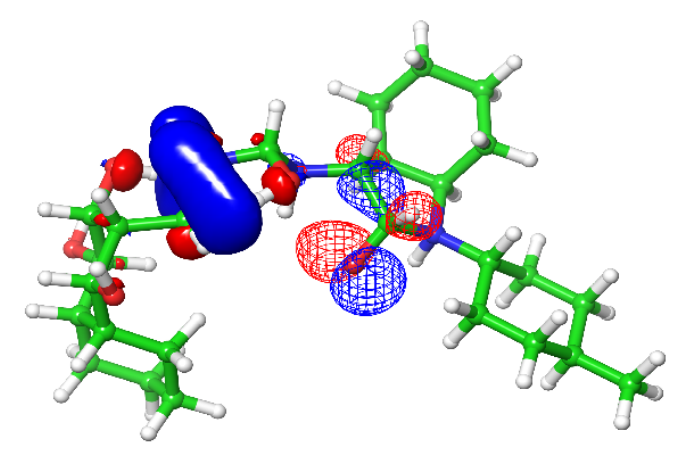

Compound 28 b)

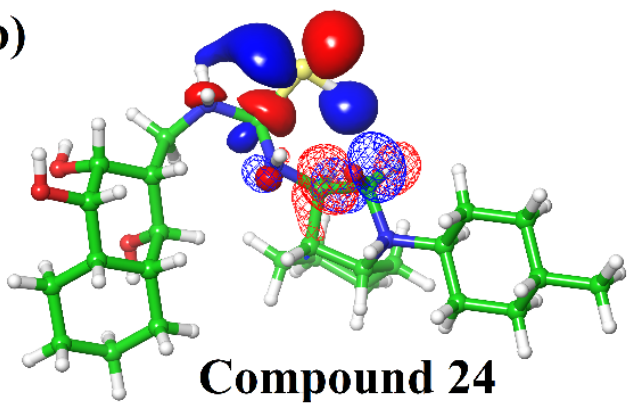

d)

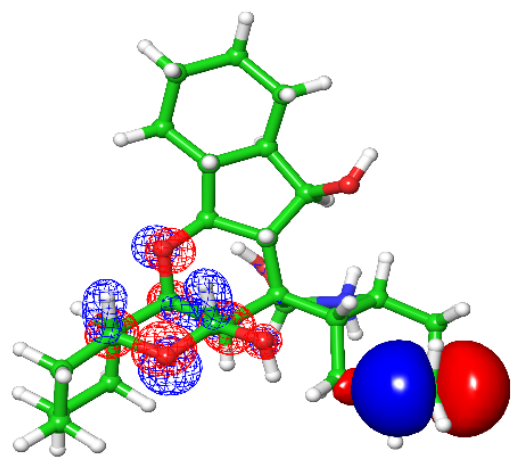

Compound 38

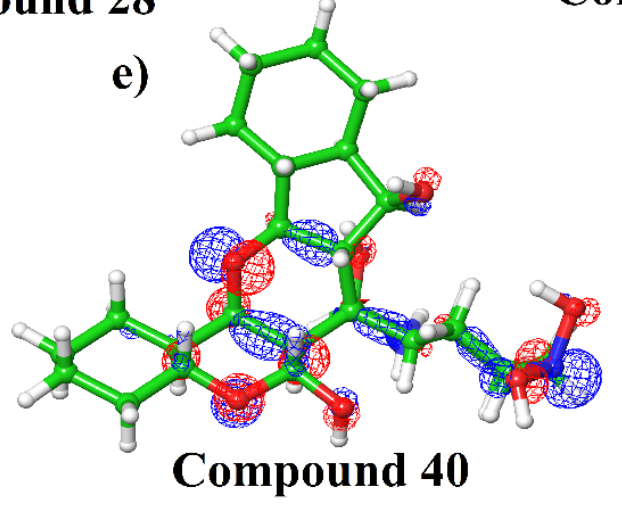

Figure 10. The HOMO and LUMO counter map analysis of top five compounds (a-e). The mesh densities denote the HOMO regions and the solid densities denote the LUMO region in the compounds. 
